\title{
Contribution of spermatozoal centrosomes to the microtubule-organizing centre in Antarctic minke whale (Balaenoptera bonaerensis)
}

\author{
Toshihiro Kobayashi ${ }^{1}$, Kazue Amemiya ${ }^{1}$,Kana Takeuchi ${ }^{1}$,Tomomi Tsujioka ${ }^{1}$, Keiichiro Tominaga ${ }^{2}$, \\ Masumi Hirabayashi ${ }^{3}$, Hajime Ishikawa ${ }^{4}$, Yutaka Fukui ${ }^{5}$ and Shinichi Hochi ${ }^{1}$ \\ Shinshu University, Hyogo Prefectural Institute for Agriculture, Forestry and Fisheries, National Institute for Physiological \\ Sciences, The Institute of Cetacean Research, and Obihiro University of Agriculture and Veterinary Medicine, Japan
}

Date submitted: 22.07.05. Date accepted 31.08.05

\section{Summary}

Using an interspecies microinsemination assay with bovine oocytes, it was examined whether centrosomes of Antarctic minke whale spermatozoa function as the microtubule-organizing centre (MTOC). Bull and rat spermatozoa were used as positive and negative controls, respectively. Vitrified-warmed bovine mature oocytes were subjected to immunostaining against $\alpha$-tubulin $4-6 \mathrm{~h}$ after intracytoplasmic injection (ICSI) of $5 \mathrm{mM}$ dithiothreitol-treated spermatozoa. Aster formation occurred from whale spermatozoa (33\%) and bull spermatozoa (33\%), but very little from rat spermatozoa (3\%). Activation treatment for the microinseminated oocytes with $7 \%$ ethanol $+2 \mathrm{mM} 6$-dimethylaminopurine resulted in a similar proportion of oocytes forming a whale sperm aster ( $35 \%$ vs $27 \%$ in the non-treated group; $4 \mathrm{~h}$ after ICSI) but a significantly larger aster (ratio of aster diameter to oocyte diameter, 0.57 vs 0.30 in the non-treated group). These results indicate that the centrosome introduced into bovine oocytes by whale spermatozoa contributes to the MTOC and that assembly of the microtubule network is promoted by oocyte activation.

Keywords: Interspecies ICSI, Minke whale, Microtubule-organizing centre (MTOC), Sperm aster

\section{Introduction}

A centrosome is composed from a pair of centrioles surrounded by pericentriolar materials such as $\gamma$-tubulin, centrin and pericentrin. Since an interphase network of microtubules and the mitotic bipolar spindle are

All correspondence to: Shinichi Hochi, Faculty of Textile Science and Technology, Shinshu University, Ueda, Nagano 386-8567, Japan. Tel: +81268 21 5350. Fax: +81 268215830 . e-mail: shochi@shinshu-u.ac.jp

${ }^{1}$ Faculty of Textile Science and Technology, Shinshu University, Ueda, Nagano 386-8567, Japan.

${ }^{2}$ Hyogo Prefectural Institute for Agriculture, Forestry and Fisheries, Kasai, Hyogo 679-0198, Japan.

${ }^{3}$ National Institute for Physiological Sciences, Okazaki, Aichi 444-8787, Japan.

${ }^{4}$ The Institute of Cetacean Research, Chuo-ku, Tokyo 1040055, Japan.

${ }^{5}$ Obihiro University of Agriculture and Veterinary Medicine, Obihiro, Hokkaido 080-8555, Japan. nucleated from the centrosome, the centrosome is considered as the microtubule-organizing centre (MTOC) (Glover et al., 1993). During fertilization in mammals, the centrosome introduced into the oocyte by the spermatozoon plays a critical role in assembly of the microtubule network that brings both male and female pronuclei to the center of the newly formed zygote, as reported in human (Simerly et al., 1995), rhesus monkey (Hewitson et al., 1996), rabbit (Pinto-Correia et al., 1994) and cattle (Navara et al., 1994). On the other hand, the paternal inheritance of MTOC does not occur in mouse (Schatten et al., 1985, 1986) and rat (Woolley \& Fawcett, 1973) and the microtubule network developed from multiple cytoplasmic asters, instead of a single sperm aster, is involved in the migration of pronuclei. Rodent spermatozoa are lacking centrioles and the majority of pericentriolar proteins after spermiogenesis (Woolley \& Fawcett, 1973; Manandhar et al., 1998, 1999).

Study of gamete interaction during fertilization is very limited in the whale. Only the early cleavages of 
minke whale oocytes after in vitro fertilization (IVF) and intracytoplasmic sperm injection (ICSI) have been reported (Fukui et al., 1997; Asada et al., 2001a, b) and it is unknown whether the paternal centrosomes contribute to the MTOC in this species. An interspecies microinsemination assay using bovine oocytes (Nakamura et al., 2001, 2002; Terada et al., 2002) or rabbit oocytes (Tarada et al., 2002, 2004) has been demonstrated to be a useful tool for evaluating the functional integrity of centrosomes in human spermatozoa. Therefore in the present study, aster formation from Antarctic whale spermatozoa microinseminated into bovine oocytes was examined.

\section{Materials and methods}

\section{Experimental design}

Mature spermatozoa from minke whale, bull and rat were pretreated with the reducing agent dithiothreitol (DTT) and were microinjected into in vitro matured bovine oocytes. Formation of the sperm aster was evaluated $4-6 \mathrm{~h}$ after interspecies ICSI. Bull and rat spermatozoa were used as positive and negative controls, respectively (Manandhar et al., 2005). Furthermore, the effect of oocyte activation treatment with ethanol and 6-dimethylaminopurine (6-DMAP) on the formation of the whale sperm aster and the subsequent development of the MTOC network was examined $4 \mathrm{~h}$ after ICSI. The Japanese Whale Research Program under Special Permit in the Antarctic Sea, conducted under Article VIII of the International Convention for the Regulation of Whaling (ICRW), allowed us to use deferent duct samples from Antarctic minke whales (Balaenoptera bonaerensis) killed by the most humane method.

\section{Preparation of bovine oocytes}

Cumulus-oocyte complexes (COCs) were recovered from abattoir-derived bovine ovaries and cultured in TCM199 medium (Gibco-BRL, Grand Island, NY) supplemented with $5 \%(\mathrm{v} / \mathrm{v})$ fetal bovine serum (FBS; Equitech Bio, Ingram, TX), $0.002 \mathrm{AU} / \mathrm{ml} \mathrm{FSH} \mathrm{(Antrin;}$ Denka Pharmaceutical, Kawasaki, Japan) and $1 \mu \mathrm{g} / \mathrm{ml}$ estradiol-17 $\beta$ (Sigma Chemical, St Louis, MO) for $22 \mathrm{~h}$ at $38.5{ }^{\circ} \mathrm{C}$ in $5 \% \mathrm{CO}_{2}$ in air, at a density of $30-50 \mathrm{COCs}$ per $750 \mu \mathrm{l}$ of medium under $250 \mu \mathrm{l}$ of mineral oil in 4-well dish (Tominaga et al., 2000). After maturation culture, oocytes were freed from cumulus cells by vortex-mixing and pipetting, and only oocytes which had extruded the first polar body were cryopreserved according to the method described by Tominaga et al. (2005) with a few modifications. Briefly, groups of five oocytes were exposed to $3 \%(\mathrm{v} / \mathrm{v})$ ethylene glycol (EG; Wako Pure Chemical Industries, Osaka, Japan) and $20 \%$ FBS in TCM199 for $12-15 \mathrm{~min}$ at $37^{\circ} \mathrm{C}$ and then transferred to a vitrification solution composed of 31\% EG, 1.0 M sucrose (Wako) and 20\% FBS in TCM199 at the same temperature. Within 30-45 s, oocytes with $0.6 \mu \mathrm{l}$ of the vitrification solution were aspirated into a gel-loading tip (BM Equipment, Tokyo, Japan) and then plunged into liquid nitrogen and stored until use. The oocytes were warmed by immersing the gel-loading tip in $1.0 \mathrm{M}$ sucrose solution for $3 \mathrm{~min}$, and then transferred into TCM199 + 20\% FBS via $0.5 \mathrm{M}$ sucrose solution at 5 min intervals. After washing three times, the oocytes were incubated in $100 \mu \mathrm{l}$ of TCM199 $+20 \%$ FBS under mineral oil for $2 \mathrm{~h}$ at $38.5^{\circ} \mathrm{C}$ in $5 \% \mathrm{CO}_{2}$ in air.

\section{Preparation of bull, rat and whale spermatozoa}

Ejaculated spermatozoa from a Japanese Black bull were derived from commercially available semen frozen in an egg yolk-Tris buffer/glycerol solution in $0.5 \mathrm{ml}$ plastic straws. The samples were thawed in a water bath at $35^{\circ} \mathrm{C}$ and washed twice by centrifugation at $490 \mathrm{~g}$ for $5 \mathrm{~min}$ with Brackett \& Oliphant (BO) solution (Brackett \& Oliphant, 1975). The sperm suspension was cryopreserved again at $-20^{\circ} \mathrm{C}$.

Cauda epididymal spermatozoa from a Wistar rat (Charles River Japan, Kanagawa, Japan) were suspended at $4^{\circ} \mathrm{C}$ in Dulbecco's phosphate-buffered saline (PBS) containing $5.6 \mathrm{mM}$ glucose, $5.4 \mathrm{mM}$ sodium lactate and $0.1 \%$ polyvinylpyrrolidone (PVP; $360 \mathrm{kDa}$; Sigma), hereafter defined as GL-PBS. The spermatozoa were exposed to $7.5 \%$ glycerol and $7.5 \%$ FBS in the GL-PBS, and then cryopreserved in $1.0 \mathrm{ml}$ cryotubes according to the method described by Ogura et al. (1996). The samples were thawed in a water bath at $20^{\circ} \mathrm{C}$ and washed twice by centrifugation at $200 \mathrm{~g}$ for 5 min with GL-PBS. The sperm suspension was cryopreserved at $-20^{\circ} \mathrm{C}$.

Spermatozoa from an Antarctic minke whale were recovered by squeezing the deferent ducts downstream, and were frozen in an egg yolk-Tris buffer/ glycerol solution in $1.0 \mathrm{ml}$ Eppendorf tubes (Mogoe et al., 1998). The samples were thawed in a water bath at $37^{\circ} \mathrm{C}$ and washed twice by centrifugation at $200 \mathrm{~g}$ for $5 \mathrm{~min}$ with GL-PBS. The sperm suspension was cryopreserved at $-20^{\circ} \mathrm{C}$.

\section{Interspecies ICSI}

The cryopreserved sperm samples were thawed in a water bath at $37^{\circ} \mathrm{C}$. An aliquot of the sperm suspension was treated with $5 \mathrm{mM} \mathrm{DTT} \mathrm{in} \mathrm{BO} \mathrm{solution} \mathrm{for} \mathrm{5-20} \mathrm{min}$ at ambient temperature $\left(23 \pm 2{ }^{\circ} \mathrm{C}\right)$, and then washed twice by centrifugation for $5 \mathrm{~min}$ at $490 \mathrm{~g}$ with $22 \mathrm{mM}$ Hepes-buffered BO solution. Immediately before the interspecies ICSI, the sperm suspension was treated with 10\% PVP in M2 medium (Quinn et al., 1982), and a single whole spermatozoon was injected into a bovine 
Table 1 Sperm aster formation in bovine oocytes injected with bull, rat and whale spermatozoa

\begin{tabular}{|c|c|c|c|c|c|c|c|}
\hline \multirow{3}{*}{$\begin{array}{l}\text { Origin of } \\
\text { spermatozoa }\end{array}$} & \multicolumn{7}{|c|}{ No. $(\%)$ of oocytes } \\
\hline & \multirow[b]{2}{*}{ Examined } & \multirow[b]{2}{*}{$\mathrm{ACT} / \mathrm{SA}$} & \multirow[b]{2}{*}{ Non-ACT/SA } & \multirow[b]{2}{*}{ ACT/Non-SA } & \multirow{2}{*}{$\begin{array}{c}\text { Non-ACT/ } \\
\text { Non-SA }\end{array}$} & \multicolumn{2}{|c|}{ Subtotal } \\
\hline & & & & & & SA & $\mathrm{ACT}$ \\
\hline Bull & 33 & $6(18)$ & $5(15)$ & $1(3)$ & $21(64)$ & $11(33)^{b}$ & $7(21)$ \\
\hline Rat & 31 & $1(3)$ & 0 & $4(13)$ & $26(84)$ & $1(3)^{a}$ & $5(16)$ \\
\hline Whale & 30 & $2(7)$ & $8(27)$ & $3(10)$ & $17(57)$ & $10(33)^{b}$ & $5(17)$ \\
\hline
\end{tabular}

ACT, activated; SA, sperm aster formed.

${ }^{a, b}$ Different superscripts within subtotal columns denote a significant difference $(p<0.05)$.

oocyte with a blunt-ended injection pipette using a piezo-impact driving unit (PMM-140FU; Prime Tech, Ibaraki, Japan) with a pulse controller (PMAS-CT140; Prime Tech). The ICSI oocytes were cultured for $4-6 \mathrm{~h}$ in $25 \mathrm{mM}$ Hepes-buffered TCM199 + $10 \%$ FBS at $38.5^{\circ} \mathrm{C}$ in $5 \% \mathrm{CO}_{2}$ in air. Some of the bovine oocytes injected with whale spermatozoa were activated by combined treatment with 7\% (v/v) ethanol (Wako) in TCM199 for 5 min and $2 \mathrm{mM}$ 6-DMAP (Sigma) in Hepes-buffered TCM199 $+10 \%$ FBS for $4 \mathrm{~h}$ at $38.5{ }^{\circ} \mathrm{C}$ in $5 \% \mathrm{CO}_{2}$ in air. Oocytes progressing beyond the metaphase II stage were considered to be activated.

\section{Evaluation of the sperm aster}

The ICSI oocytes were fixed and labelled with microtubule-specific antibody, according to the methods described by Simerly \& Schatten (1993) and Nakamura et al. (2001). Briefly, oocytes were extracted for $15 \mathrm{~min}$ by buffer M $(25 \%$ glycerol, $50 \mathrm{mM} \mathrm{KCl}$, $0.5 \mathrm{mM} \mathrm{MgCl}_{2}, 0.1 \mathrm{mM}$ EDTA, $1 \mathrm{mM}$ EGTA, $50 \mathrm{mM}$ imidazole hydrochloride, and $1 \mathrm{mM}$ 2-mercaptoethanol, $\mathrm{pH}$ 6.8) containing $5 \%(\mathrm{v} / \mathrm{v})$ methanol and $1 \%(\mathrm{v} / \mathrm{v})$ Triton X-100 detergent, after zonae pellucidae had been removed with $0.75 \%$ protease (Sigma) in M2 medium. The oocytes were then fixed with cold methanol for $10 \mathrm{~min}$ and permeabilized overnight in $0.1 \mathrm{M}$ PBS containing $0.1 \%(\mathrm{v} / \mathrm{v})$ Triton X-100. Microtubules were labelled with a monoclonal antibody against $\alpha$-tubulin (T5168; diluted 1:4000; Sigma). The primary antibodies were detected by fluorescein isothiocyanate (FITC)-conjugated goat anti-mouse IgG (F1010; diluted 1:512; Sigma). Nuclear DNA was visualized by staining with $2.5 \mu \mathrm{g} / \mathrm{ml}$ DAPI (Sigma). The preparations were mounted with coverslips in $100 \mathrm{mg} / \mathrm{ml} \mathrm{1,4-dia-}$ zabicyclo[2.2.2]octane (DABCO; Sigma) dissolved in glycerol:PBS $(9: 1 ; \mathrm{v} / \mathrm{v})$, and examined using a conventional epifluorescence microscope. Digital images were processed using Adobe Photoshop software (Adobe Systems, Mountain View, CA). Aster size was calculated as the mean diameter of the sperm aster measured at its widest and highest points.

\section{Statistical analysis}

All experiments were repeated at least five times. Data for sperm aster formation and oocyte activation were analysed by Fisher's exact probability test between the experimental group (whale) and control groups (bull and rat), and between the activation treatment group and the non-treated group. Aster size was recalculated as a ratio to the oocyte diameter and the proportional data were compared by $t$-test after arc-sin transformation. A value of $p<0.05$ was chosen as an indication of statistical significance.

\section{Results}

The proportions of bovine oocytes forming a sperm aster are shown in Table 1. Eleven (33\%) of the 33 oocytes injected with bull spermatozoa had a microtubule network originated from the sperm heads, and six of them progressed to the anaphase stage (Fig. 1A). No sign of sperm aster formation and meiosis resumption was found in the 31 oocytes injected with rat spermatozoa (Fig. 1B), with the exception of one oocyte (Fig. 1B'). Of the 30 oocytes injected with whale sperm, $10(33 \%)$ had a sign of microtubule extension (Fig. 1C), but only 2 of the 10 oocytes had progressed beyond the metaphase II stage.

The effect of activation treatment (ethanol +6DMAP) in bovine oocytes on aster formation originated from whale spermatozoa is shown in Table 2. A lower proportion of bovine oocytes injected with whale spermatozoa resumed meiosis without the aid of chemical activation treatment ( $23 \%$ vs $97 \%$ in the treatment group). The proportion of oocytes forming a sperm aster was not significantly improved by the activation treatment ( $35 \%$ vs $27 \%$ in non-treated group). However, the diameter of the whale sperm aster in the 

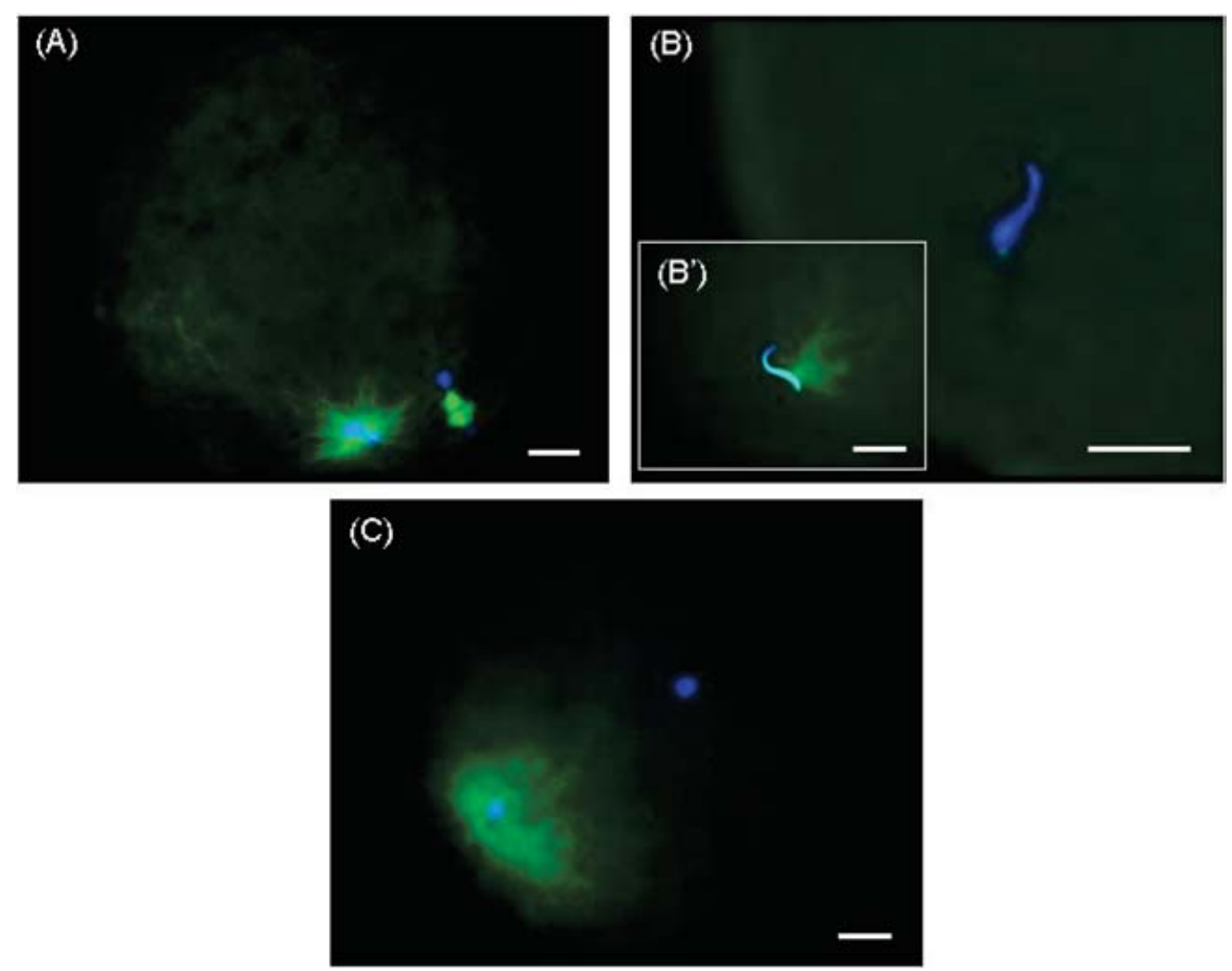

Figure 1 The $\alpha$-tubulin-immunostained (green) and DAPI-stained (blue) bovine oocytes after injection with bull, rat and whale spermatozoa. (A) Bull sperm aster and anaphase II plate. (B) Rat sperm head without any sign of aster formation, and ( $\left.B^{\prime}\right)$ a figure of rat sperm aster. $(C)$ An example of whale sperm aster. Scale bar represents $10 \mu \mathrm{m}$.

Table 2 Effect of activation treatment of bovine oocytes on formation of aster originated from whale spermatozoa

\begin{tabular}{lcccccrr}
\hline & \multicolumn{4}{c}{ No. $(\%)$ of oocytes } \\
\cline { 2 - 8 } $\begin{array}{l}\text { Activation } \\
\text { treatment }\end{array}$ & Examined & ACT/SA & Non-ACT/SA & ACT/Non-SA & $\begin{array}{c}\text { Non-ACT/ } \\
\text { Non-SA }\end{array}$ & SA & ACT \\
\hline- & 30 & $3(10)$ & $5(17)$ & $4(13)$ & $18(60)$ & $8(26)$ & $7(23)^{a}$ \\
+ & 31 & $11(35)$ & 0 & $19(61)$ & $1(3)$ & $11(35)$ & $30(97)^{b}$ \\
\hline
\end{tabular}

ACT, activated; SA, sperm aster formed.

${ }^{a, b}$ Different superscripts within subtotal columns denote a significant difference $(p<0.05)$.

treated oocytes was significantly larger than that in the non-treated oocytes (Fig. 2).

\section{Discussion}

The centrosome in whale spermatozoa was capable of contributing to the MTOC, when assessed by interspecies microinsemination with bovine oocytes (Table 1). Rodent/non-rodents interspecies assays by IVF and ICSI have been used to evaluate sperm capacitation status (e.g. sperm penetration assay into zona-free hamster oocytes; Hanada \& Chang, 1972) and sperm-borne oocyte activating factor (SOAF) (e.g. mouse oocyte activation assay; Rybouchkin et al., 1995), respectively. In the latter (Rybouchkin et al., 1995) and other studies (Lee et al., 1996), chromosomes of human spermatozoa have also been visualized in mouse oocytes. However, such rodent oocytes are not valid for assessing the functional integrity of the non-rodent sperm centrosome, because the cytoplasmic aster(s), instead of the sperm aster, play a critical role in the migration of pronuclei in the rodent zygotes (Schatten 


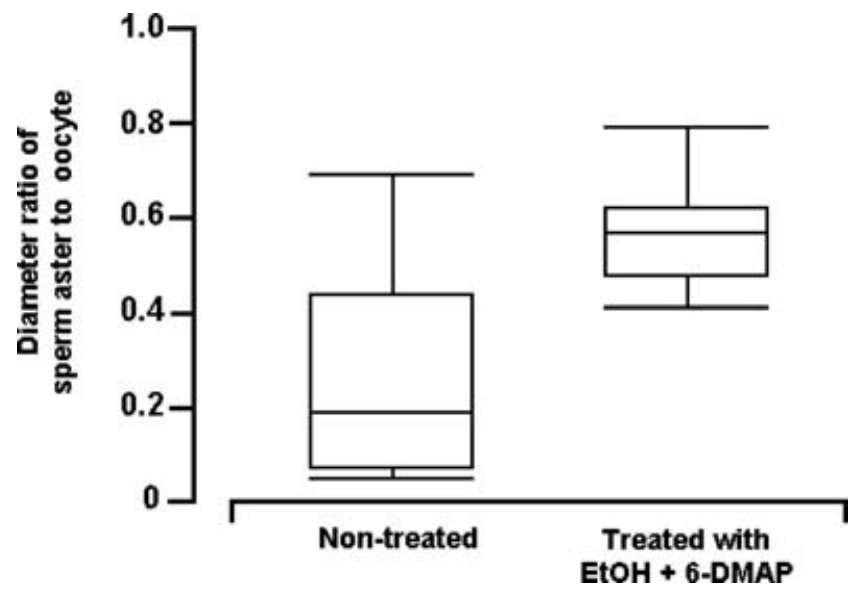

Figure 2 Size of whale sperm aster observed in artificially activated and non-treated bovine oocytes. The ratios of aster diameter to oocyte diameter were box-plotted.

et al., 1985, 1986). In fact, normal human spermatozoa did not organize the sperm aster in hamster oocytes (Hewitson et al., 1997). Therefore, microinsemination into oocytes from rabbit (Terada et al., 2002, 2004) or cattle (Nakamura et al., 2001, 2002; Terada et al., 2002) has been proposed to assess the dysfunction of the human sperm centrosome in relation to male infertility. Interspecies ICSI using bovine oocytes would be more convenient to achieve, because in vitro matured bovine oocytes are easy to prepare provided abattoirderived ovaries are available. Nevertheless, the poor availability of bovine ovaries in our laboratory and a daily limit on the number of treated oocytes (less than 10) forced us to use the cryopreserved oocytes in the present study. The gel-loading tip vitrification procedure, characterized by an ultra-rapid cooling rate (Tominaga \& Hamada, 2001), was found to be a promising method for cryopreservation of bovine oocytes after some modifications (Tominaga et al., 2005). However, an adverse effect of using vitrified oocytes and/or re-frozen spermatozoa on sperm aster formation cannot be ruled out.

When bull spermatozoa pretreated with DTT were microinjected into bovine oocytes, a partial decondensation of the sperm nucleus was induced (Rho et al., 1998), probably because the sperm nuclear structure condensed and stabilized by disulfide bonds (Calvin \& Bedford, 1971; Calvin et al., 1973; Marushige \& Marushige, 1975) was changed by the DTT treatment. Pretreatment of spermatozoa with DTT was employed in the present study because it improved the proportion of oocytes with a sperm aster-derived microtubule network in a preliminary experiment on bovine ICSI (data not shown). The DTT not only destabilizes nuclear packaging in the sperm head but also organizes $\gamma$-tubulin in the sperm centrosome, from which microtubules are nucleated (Schatten, 1994). The conformational change induced by reducing the disulfide bonds would facilitate the $\gamma$-tubulin to access the microtubule components ( $\alpha$ - and $\beta$-tubulin) present in the ooplasm. In the present study, bull and rat spermatozoa were used as positive and negative controls, respectively, because the fate of sperm centrioles during spermiogenesis shows a remarkable contrast between the two species (Manandhar et al., 2005). Although none of the bovine oocytes injected with rat spermatozoa was expected to form a sperm aster, one rat sperm aster was observed. A possible explanation is that the aster may be nucleated from pericentriolar proteins remaining after spermiogenesis. A bovine cyto-aster was not observed during at least $4 \mathrm{~h}$ culture of 34 non-treated and 32 ethanol/6-DMAP-treated oocytes without sperm injection (data not shown).

Microinsemination of whale spermatozoa resulted in sperm aster formation in some bovine oocytes, but only a few oocytes resumed meiosis $4-6 \mathrm{~h}$ after ICSI (Table 1). However, we found that whale spermatozoa acquired a sufficient level of SOAF activity during spermiogenesis, assessed by meiosis resumption of microinseminated mouse oocytes (Amemiya et al., 2004). It may take into account this contradiction that the whale SOAF is unable to trigger the cascade for activation of bovine oocytes three times larger in volume than mouse oocytes. The post-ICSI treatment of bovine oocytes with ethanol and 6-DMAP did not improve the proportion of oocytes forming a whale sperm aster (Table 2), but contributed to development of a microtubule network (Fig. 2). In bovine ICSI, however, it seems that the sperm aster formation rate is improved not only by treatment of the spermatozoa with DTT but also by the activation with ethanol of injected oocytes (Dr T. Horiuchi, Hiroshima Prefectural University, Japan, personal communication). Fujinami et al. (2005) observed that following treatment with 7\% ethanol, $58 \%$ of bovine oocytes formed a sperm aster $6 \mathrm{~h}$ after ICSI, versus $12 \%$ without ethanol treatment. Wei \& Fukui (2000) reported that bovine oocytes injected with whale spermatozoa cleaved only when the oocytes were activated with $7 \%$ ethanol $30 \mathrm{~min}$ before and $1 \mathrm{~h}$ after ICSI. To date, production of whale blastocysts by IVF or ICSI has not been successful (Fukui et al., 1997; Asada et al., 2001a, b). Since whale oocytes are similar to bovine oocytes in size and appearance (Fukui et al., 1997), the artificial activation treatment for ICSI oocytes, as well as the pretreatment of sperm with DTT and the microinjection of an intact head-midpiece junction site, may be essential for the production of developmentally competent whale zygotes.

In conclusion, mature spermatozoa from the Antarctic minke whale have centrosomes capable of contributing to the MTOC, as assessed by interspecies ICSI using bovine oocytes. The SOAF activity of whale 
spermatozoa was not sufficient to resume meiosis of bovine oocytes. The development of the microtubule network was promoted when the ICSI oocytes were activated with a combined treatment of ethanol and 6DMAP. This fundamental knowledge and insight may be useful for the production of whale embryos by ICSI.

\section{Acknowledgements}

The authors thank the Captain and crew of the research base ship Nisshin-maru for their help in obtaining whale spermatozoa. This work was supported in part by a Grant-in-Aid for the 21st Century COE program from the Ministry of Education, Culture, Sports, Science and Technology of Japan.

\section{References}

Amemiya, K., Iwanami, Y., Kobayashi, T., Terao, T., Fukui, Y., Ishikawa, H., Ohsumi, S., Hirabayashi, M. \& Hochi, S. (2004). Acquirement of oocyte-activating factor in Antarctic minke whale (Balaenoptera bonaerensis) spermatogenic cells, assessed by meiosis resumption of microinseminated mouse oocytes. J. Mamm. Ova Res. 21, 149-56.

Asada, M., Tetsuka, M., Ishikawa, H., Ohsumi, S. \& Fukui, Y. (2001a). Improvement of in vitro maturation, fertilization and development of minke whale (Balaenoptera acutorostrata) oocytes. Theriogenology 56, 521-33.

Asada, M., Wei, H., Nagayama, R., Tetsuka, M., Ishikawa, H., Ohsumi, S. \& Fukui, Y. (2001b). An attempt at intracytoplasmic sperm injection of frozen-thawed minke whale (Balaenoptera bonaerensis) oocytes. Zygote 9, 299307.

Brackett, B.G. \& Oliphant, G. (1975). Capacitation of rabbit spermatozoa in vitro. Biol. Reprod. 12, 260-74.

Calvin, H.I. \& Bedford, J.M. (1971). Formation of disulfide bonds in the nucleus and accessory structures of mammalian spermatozoa during maturation in the epididymis. J. Reprod. Fertil. Suppl. 13, 65-75.

Calvin, H.I., Yu, C.C. \& Bedford, J.M. (1973) Effects of epididymal maturation, zinc and copper on the reactive sulfhydryl content of structural elements in rat spermatozoa. Exp. Cell Res. 81, 333-41.

Fujinami, N., Hosoi, Y., Kato, H., Mitani, T., Matsumoto, K., Saeki, K. \& Iritani, A. (2005) Effects of ethanol treatment after intracytoplasmic sperm injection (ICSI) on sperm aster formation and the microtubule organization of bovine oocytes (abstract). Reprod. Fertil. Dev. 17, 307.

Fukui, Y., Mogoe, T., Ishikawa, H. \& Ohsumi, S. (1997). In vitro fertilization of in vitro matured minke whale (Balaenoptera acutorostrata) follicular oocytes. Mar. Mamm. Sci. 13, 395404.

Glover, D.M, Gonzalez, C. \& Raff, J.W. (1993). The centrosome. Sci. Am. 268, 62-8.

Hanada, A. \& Chang, M.C. (1972). Penetration of zona-free eggs by spermatozoa of different species. Biol. Reprod. 6, 300-9.
Hewitson, L.C., Simerly, C.R., Tengowski, M.W., Sutovsky, P., Navara, C.S., Haavisto, A.J. \& Schatten, G. (1996). Microtubule and chromatin configurations during rhesus intracytoplasmic sperm injection: successes and failures. Biol. Reprod. 55, 271-80.

Hewitson, L., Haavisto, A., Simerly, C., Jones, J. \& Schatten, G. (1997). Microtubule organization and chromatin configurations in hamster oocytes during fertilization and parthenogenetic activation, and after insemination with human sperm. Biol. Reprod. 57, 967-75.

Lee, J.D., Kamiguchi, Y. \& Yanagimachi, R. (1996). Analysis of chromosome constitution of human spermatozoa with normal and aberrant head morphologies after injection into mouse oocytes. Hum. Reprod. 11, 1942-6.

Manandhar, G., Sutovsky, P., Joshi, H.C., Stearns, T. \& Schatten, G. (1998). Centrosome reduction during mouse spermiogenesis. Dev. Biol. 203, 424-34.

Manandhar, G., Simerly, C., Salisbury, J.L. \& Schatten, G. (1999). Centriole and centrin degeneration during mouse spermiogenesis. Cell Motil. Cytoskeleton 43, 137-44.

Manandhar, G., Schatten, H. \& Sutovsky, P. (2005). Centrosome reduction during gametogenesis and its significance. Biol. Reprod. 72, 2-13.

Marushige, Y. \& Marushige, K. (1975). Transformation of sperm histone during formation and maturation of rat spermatozoa.J. Biol. Chem. 250, 39-45.

Mogoe, T., Fukui, Y., Ishikawa, H. \& Ohsumi, S. (1998). Effect of diluent composition and temperature on motility and viability after liquid storage and cryopreservation of minke whale (Balaenoptera bonaerensis) spermatozoon. Mar. Mamm. Sci. 14, 854-60.

Nakamura, S., Terada, Y., Horiuchi, T., Emuta, C., Murakami, T., Yaegashi, N. \& Okamura, K. (2001). Human sperm aster formation and pronuclear decondensation in bovine eggs following intracytoplasmic sperm injection using a Piezodriven pipette: a novel assay for human sperm centrosomal function. Biol. Reprod. 65, 1359-63.

Nakamura, S., Terada, Y., Horiuchi, T., Emuta, C., Murakami, T., Yaegashi, N. \& Okamura, K. (2002). Analysis of the human sperm centrosomal function and the oocyte activation ability in a case of globozoospermia, by ICSI into bovine oocytes. Hum. Reprod. 17, 2930-4.

Navara, C.S., First, N.L. \& Schatten, G. (1996). Phenotypic variations among paternal centrosomes expressed within the zygote as disparate microtubule lengths and sperm aster organization: correlations between centrosome activity and developmental success. Proc. Natl. Acad. Sci. USA 93, 5384-8.

Ogura, A., Matsuda, J., Asano, T., Suzuki, O. \& Yanagimachi, R. (1996). Mouse oocytes injected with cryopreserved round spermatids can develop into normal offspring. $J$. Assist. Reprod. Genet. 5, 431-4.

Pinto-Correia, C., Poccia, D.L., Chang, T. \& Robl, J.M. (1994). Dephosphorylation of sperm midpiece antigens initiates aster formation in rabbit oocytes. Proc. Natl. Acad. Sci. USA 91, 7894-8.

Quinn, P., Barros, C. \& Whittingham, D.G. (1982). Preservation of hamster oocytes to assay the fertilizing capacity of human spermatozoa. J. Reprod. Fertil. 66, 161-8.

Rho, G.J., Kawarsky, S., Johnson, W.H., Kochhar, K. \& Betteridge, K.J. (1998). Sperm and oocyte treatments to 
improve the formation of male and female pronuclei and subsequent development following intracytoplasmic sperm injection into bovine oocytes. Biol. Reprod. 59, 918-24.

Rybouchkin, A., Dozortsev, D., De Sutter, P., Quin, C. \& Dhont, M. (1995). Intracytoplasmic injection of human spermatozoa into mouse oocytes: a useful model to investigate the oocyte-activating capacity and the karyotype of human spermatozoa. Hum. Reprod. 10, 1130-5.

Schatten, G. (1994) The centrosome and its mode of inheritance: the reduction of the centrosome during gametogenesis and its restoration during fertilization. Dev. Biol. 165, 299-335.

Schatten, G., Simerly, C. \& Schatten, H. (1985). Microtubule configurations during fertilization, mitosis, and early development in the mouse and the requirement for egg microtubule-mediated motility during mammalian fertilization. Proc. Natl. Acad. Sci. USA 82, 4152-6.

Schatten, H., Schatten, G., Mazia, D., Balczon, R. \& Simerly, C. (1986). Behavior of centrosomes during fertilization and cell division in mouse oocytes and in sea urchin eggs. Proc. Natl. Acad. Sci. USA 83, 105-9.

Simerly, C. \& Schatten, G. (1993). Techniques for localization of specific molecules in oocytes and embryos. Methods Enzymol. 225, 516-53.

Simerly, C., Wu, G.J., Zoran, S., Ord, T., Rawlins, R., Jones, J., Navara, C., Gerrity, M., Rinehart, J. \& Binor, Z. (1995). The paternal inheritance of the centrosome, the cell's microtubule-organizing center, in humans, and the implications for infertility. Nat. Med. 1, 47-52.
Terada, Y., Nakamura, S., Hewitson, L., Simerly, C., Horiuchi, T., Murakami, T., Okamura, K. \& Schatten, G. (2002). Human sperm aster formation after intracytoplasmic sperm injection with rabbit and bovine eggs. Fertil. Steril. 77, 1283-4.

Terada, Y., Nakamura, S., Simerly, C., Hewitson, L., Murakami, T., Yaegashi, N., Okamura, K. \& Schatten, G. (2004). Centrosomal function assessment in human sperm using heterologous ICSI with rabbit eggs: a new male factor infertility assay. Mol. Reprod. Dev. 67, 360-5.

Tominaga, K. \& Hamada, Y. (2001). Gel-Loading tip as container for vitrification of in vitro-produced bovine embryos. J. Reprod. Dev. 47, 267-73.

Tominaga, K., Shimizu, M., Ooyama, S. \& Izaike, Y. (2000). Effect of lipid polarization by centrifugation at different developmental stages on post-thaw survival of bovine in vitro produced 16-cell embryos. Theriogenology 53, 166980.

Tominaga, K., Hamada, Y. \& Hochi, S. (2005). Gel-Loading tip vitrification of in vitro-matured bovine oocytes and subsequent embryo production by IVF and nuclear transfer. J. Mamm. Ova Res. 22, 178-84.

Wei, H. \& Fukui, Y. (2000). Fertilisability of ovine, bovine or minke whale (Balaenoptera acutorostrata) spermatozoa intracytoplasmically injected into bovine oocytes. Zygote 8, 267-74.

Woolley, D.M. \& Fawcett, D.W. (1973). The degeneration and disappearance of the centrioles during the development of the rat spermatozoon. Anat. Rec. 177, 289-302. 